DOI: $10.2478 /$ awutm-2013-0003<smiles>CC1=CC=C1</smiles>

VERSITA
Analele Universitătii de Vest, Timişoara

Seria Matematică - Informatică LI, 1, (2013), 37- 45

\title{
Radius Problems for Certain Classes of Analytic Functions
}

Basem Aref Frasin and Jin-Lin Liu

Abstract. Let $\mathcal{P}\left(\beta_{1}, \beta_{2}, \beta_{3} ; \lambda\right)$ be a subclass of analytic functions $f(z)$ which satisfies $\frac{f(z)}{z} \neq 0 \quad(z \in \mathcal{U})$ and

$$
\left|\beta_{1}\left(\frac{z}{f(z)}\right)^{\prime \prime}+\beta_{2}\left(\frac{z}{f(z)}\right)^{\prime \prime \prime}+\beta_{3}\left(\frac{z}{f(z)}\right)^{\prime \prime \prime \prime}\right| \leq \lambda \quad(z \in \mathcal{U}),
$$

for some complex numbers $\beta_{1}, \beta_{2}, \beta_{3}$ and for some real $\lambda>0$. The object of the present paper is to obtain radius problems of $\frac{1}{\delta} f(\delta z) \in \mathcal{P}\left(\beta_{1}, \beta_{2}, \beta_{3} ; \lambda\right)$ if $f(z)$ satisfies $\operatorname{Re}\left\{\frac{1-z\left(\frac{z}{f(z)}\right)^{\prime \prime}}{1+\left(\frac{z}{f(z)}\right)^{\prime}}\right\}>\alpha$.

AMS Subject Classification (2000). 30C45.

Keywords. Analytic functions, univalent functions, CauchySchwarz inequality, radius problems.

\section{Introduction and definitions}

Let $\mathcal{A}$ denote the class of the normalized functions of the form

$$
f(z)=z+\sum_{n=2}^{\infty} a_{n} z^{n},
$$


which are analytic in the open unit disk $\mathcal{U}=\{z \in \mathbb{C}:|z|<1\}$. Further, let $\mathcal{H}(\alpha)$ be the subclass of analytic functions $f(z)$ defined by

$$
\operatorname{Re}\left\{\frac{1-z\left(\frac{z}{f(z)}\right)^{\prime \prime}}{1+\left(\frac{z}{f(z)}\right)^{\prime}}\right\}>\alpha \quad(0 \leq \alpha<1 ; z \in \mathcal{U})
$$

where $\frac{z}{f(z)}=1+\sum_{n=1}^{\infty} b_{n} z^{n}, b_{n}=\left|b_{n}\right| e^{i(n-1) \theta}$.

For a function $f(z)$ belonging to $\mathcal{A}$ is said to be in the class $\mathcal{P}\left(\beta_{1}, \beta_{2}, \beta_{3} ; \lambda\right)$ if it satisfies $\frac{f(z)}{z} \neq 0 \quad(z \in \mathcal{U})$ and

$$
\left|\beta_{1}\left(\frac{z}{f(z)}\right)^{\prime \prime}+\beta_{2}\left(\frac{z}{f(z)}\right)^{\prime \prime \prime}+\beta_{3}\left(\frac{z}{f(z)}\right)^{\prime \prime \prime \prime}\right| \leq \lambda \quad(z \in \mathcal{U}),
$$

for some complex numbers $\beta_{1}, \beta_{2}, \beta_{3}$ and for some real $\lambda>0$. Very recently, Kobashi et al. [3] have studied the class $\mathcal{P}\left(\beta_{1}, \beta_{2}, 0 ; \lambda\right)$ ( see also, [2]) and Kobashi et al. [1] have considered the class $\mathcal{P}_{4}(\lambda)=\mathcal{P}(0,0,1 ; \lambda)$ defined by

$$
\left|\left(\frac{z}{f(z)}\right)^{\prime \prime \prime \prime}\right| \leq \lambda \quad(z \in \mathcal{U})
$$

Let us consider the function $f_{\gamma}(z)$ given by $f_{\gamma}(z)=\frac{z}{(1-z)^{\gamma}} \quad(\gamma \geq 0)$. Then,we observe that

$$
\frac{f_{\gamma}(z)}{z}=\frac{1}{(1-z)^{\gamma}} \neq 0 \quad(z \in \mathcal{U})
$$

and

$$
\begin{aligned}
& \left|\beta_{1}\left(\frac{z}{f_{\gamma}(z)}\right)^{\prime \prime}+\beta_{2}\left(\frac{z}{f_{\gamma}(z)}\right)^{\prime \prime \prime}+\beta_{3}\left(\frac{z}{f_{\gamma}(z)}\right)^{\prime \prime \prime \prime}\right| \\
= & \mid \beta_{1} \gamma(\gamma-1)(1-z)^{\gamma-2}+\beta_{2} \gamma(\gamma-1)(\gamma-2)(1-z)^{\gamma-3} \\
& +\beta_{3} \gamma(\gamma-1)(\gamma-2)(\gamma-3)(1-z)^{\gamma-4} \mid \\
< & \mid \beta_{1} \gamma(\gamma-1)(2)^{\gamma-2}+\beta_{2} \gamma(\gamma-1)(\gamma-2)(2)^{\gamma-3} \\
& +\beta_{3} \gamma(\gamma-1)(\gamma-2)(\gamma-3)(2)^{\gamma-4} \mid
\end{aligned}
$$

Therefore, if $\gamma=2$, then

$$
\left|\beta_{1}\left(\frac{z}{f_{2}(z)}\right)^{\prime \prime}+\beta_{2}\left(\frac{z}{f_{2}(z)}\right)^{\prime \prime \prime}+\beta_{3}\left(\frac{z}{f_{2}(z)}\right)^{\prime \prime \prime \prime \prime}\right|<2\left|\beta_{1}\right| .
$$

This implies that $f_{2}(z) \in \mathcal{P}\left(\beta_{1}, \beta_{2}, \beta_{3} ; \lambda\right)$ for $\lambda>2\left|\beta_{1}\right|$. If $\gamma=3$, then we have 


$$
\left|\beta_{1}\left(\frac{z}{f_{3}(z)}\right)^{\prime \prime}+\beta_{2}\left(\frac{z}{f_{3}(z)}\right)^{\prime \prime \prime}+\beta_{3}\left(\frac{z}{f_{3}(z)}\right)^{\prime \prime \prime \prime}\right|<6\left(2\left|\beta_{1}\right|+\left|\beta_{2}\right|\right) .
$$

Thus, $f_{3}(z) \in \mathcal{P}\left(\beta_{1}, \beta_{2}, \beta_{3} ; \lambda\right)$ for $\lambda>6\left(2\left|\beta_{1}\right|+\left|\beta_{2}\right|\right)$. Further, if $\gamma=4$, then we have

$$
\left|\beta_{1}\left(\frac{z}{f_{4}(z)}\right)^{\prime \prime}+\beta_{2}\left(\frac{z}{f_{4}(z)}\right)^{\prime \prime \prime}+\beta_{3}\left(\frac{z}{f_{4}(z)}\right)^{\prime \prime \prime \prime}\right|<8\left(6\left|\beta_{1}\right|+6\left|\beta_{2}\right|+3\left|\beta_{3}\right|\right) .
$$

Therefore, $f_{4}(z) \in \mathcal{P}\left(\beta_{1}, \beta_{2}, \beta_{3} ; \lambda\right)$ for $\lambda>8\left(6\left|\beta_{1}\right|+6\left|\beta_{2}\right|+3\left|\beta_{3}\right|\right)$.

If we consider the function $f(z)$ given by $f(z)=\frac{z}{\sum_{k=0}^{n} z^{k}}$, then

$$
\begin{aligned}
& \left|\beta_{1}\left(\frac{z}{f(z)}\right)^{\prime \prime}+\beta_{2}\left(\frac{z}{f(z)}\right)^{\prime \prime \prime}+\beta_{3}\left(\frac{z}{f(z)}\right)^{\prime \prime \prime \prime}\right| \\
< & \left|\beta_{1}\right| \sum_{k=2}^{n} k(k-1)+\left|\beta_{2}\right| \sum_{k=3}^{n} k(k-1)(k-2)+\left|\beta_{3}\right| \sum_{k=4}^{n} k(k-1)(k-2)(k-3) \\
= & n(n+1)\left[\frac{\left|\beta_{2}\right|(n-1)}{3}+\frac{\left|\beta_{3}\right|(n-1)(n-2)}{4}+\frac{\left|\beta_{3}\right|(n-1)(n-2)(n-3)}{5}\right] .
\end{aligned}
$$

Therefore, $f(z) \in \mathcal{P}\left(\beta_{1}, \beta_{2}, \beta_{3} ; \lambda\right)$, with

$\lambda=n(n+1)\left[\frac{\left|\beta_{2}\right|(n-1)}{3}+\frac{\left|\beta_{3}\right|(n-1)(n-2)}{4}+\frac{\left|\beta_{3}\right|(n-1)(n-2)(n-3)}{5}\right]$.

\section{Radius problem for the class $\mathcal{P}\left(\beta_{1}, \beta_{2}, \beta_{3} ; \lambda\right)$}

To obtain the radius problem for the class $\mathcal{P}\left(\beta_{1}, \beta_{2}, \beta_{3} ; \lambda\right)$, we need the following lemmas.

Lemma 2.1. Let $f(z) \in \mathcal{A}$ and $\frac{z}{f(z)}=1+\sum_{n=1}^{\infty} b_{n} z^{n} \neq 0(z \in \mathcal{U})$. If $f(z)$ satisfies

$$
\sum_{n=2}^{\infty} n(n-1)\left(\left|\beta_{1}\right|+(n-2)\left|\beta_{2}\right|+(n-2)(n-3)\left|\beta_{3}\right|\right)\left|b_{n}\right| \leq \lambda
$$

for some complex numbers $\beta_{1}, \beta_{2}, \beta_{3}$ and for some real $\lambda>0$, then $f(z) \in$ $\mathcal{P}\left(\beta_{1}, \beta_{2}, \beta_{3} ; \lambda\right)$. 
Proof. We observe that

$$
\begin{aligned}
& \left|\beta_{1}\left(\frac{z}{f(z)}\right)^{\prime \prime}+\beta_{2}\left(\frac{z}{f(z)}\right)^{\prime \prime \prime}+\beta_{3}\left(\frac{z}{f(z)}\right)^{\prime \prime \prime \prime}\right| \\
& =\mid \beta_{1} \sum_{n=2}^{\infty} n(n-1) b_{n} z^{n-2}+\beta_{2} \sum_{n=2}^{\infty} n(n-1)(n-2) b_{n} z^{n-3} \\
& +\beta_{3} \sum_{n=4}^{\infty} n(n-1)(n-2)(n-3) b_{n} z^{n-4} \\
& \leq\left|\beta_{1}\right| \sum_{n=2}^{\infty} n(n-1)\left|b_{n}\right|+\left|\beta_{2}\right| \sum_{n=3}^{\infty} n(n-1)(n-2)\left|b_{n}\right|+ \\
& \left|\beta_{3}\right| \sum_{n=4}^{\infty} n(n-1)(n-2)(n-3)\left|b_{n}\right| \\
& =\sum_{n=2}^{\infty} n(n-1)\left(\left|\beta_{1}\right|+(n-2)\left|\beta_{2}\right|+(n-2)(n-3)\left|\beta_{3}\right|\right)\left|b_{n}\right|
\end{aligned}
$$

Therefore, if $f(z)$ satisfies the inequality (2.1), then $f(z) \in \mathcal{P}\left(\beta_{1}, \beta_{2}, \beta_{3} ; \lambda\right)$.

Lemma 2.2. If $f(z) \in \mathcal{H}(\alpha) ; 0 \leq \alpha<\frac{1}{1+\left|b_{1}\right|}$ and $\frac{z}{f(z)}=1+\sum_{n=1}^{\infty} b_{n} z^{n}$, $b_{n}=\left|b_{n}\right| e^{i(n-1) \theta}$, then

$$
\sum_{n=2}^{\infty} n(n-1+\alpha)\left|b_{n}\right| \leq 1-\alpha-\alpha\left|b_{1}\right|
$$

Proof. Let $f(z) \in \mathcal{H}(\alpha)$ and $\frac{z}{f(z)}=1+\sum_{n=1}^{\infty} b_{n} z^{n}, b_{n}=\left|b_{n}\right| e^{i(n-1) \theta}(n=$ $1,2, \cdots)$. Then,

$$
\begin{aligned}
\operatorname{Re}\left\{\frac{1-z\left(\frac{z}{f(z)}\right)^{\prime \prime}}{1+\left(\frac{z}{f(z)}\right)^{\prime}}\right\} & =\operatorname{Re}\left(\frac{1-\sum_{n=2}^{\infty} n(n-1) b_{n} z^{n-1}}{1+\sum_{n=1}^{\infty} n b_{n} z^{n-1}}\right) \\
& =\operatorname{Re}\left(\frac{1-\sum_{n=2}^{\infty} n(n-1)\left|b_{n}\right| e^{i(n-1) \theta} z^{n-1}}{1+\sum_{n=1}^{\infty} n\left|b_{n}\right| e^{i(n-1) \theta} z^{n-1}}\right)>\alpha \quad(z \in \mathcal{U}) .
\end{aligned}
$$


If we consider a point $z=|z| e^{-i \theta}$, then we have

$$
\frac{1-\sum_{n=2}^{\infty} n(n-1)\left|b_{n}\right||z|^{n-1}}{1+\left|b_{1}\right|+\sum_{n=2}^{\infty} n\left|b_{n}\right||z|^{n-1}}>\alpha .
$$

Letting $|z| \rightarrow 1^{-}$, we obtain the inequality (2.2), and this completes the proof.

Remark 2.1. If $f(z) \in \mathcal{H}(\alpha) ; 0 \leq \alpha<\frac{1}{1+\left|b_{1}\right|}$, then the inequality

$$
\sum_{n=2}^{\infty} n(n-1+\alpha)\left|b_{n}\right| \leq 1-\alpha-\alpha\left|b_{1}\right|
$$

implies that

$$
\sum_{n=2}^{\infty} n(n-1)\left|b_{n}\right|^{2} \leq 1-\alpha-\alpha\left|b_{1}\right|
$$

Applying the above lemmas, we derive the following theorem.

Theorem 2.3. If $f(z) \in \mathcal{H}(\alpha) ; 0 \leq \alpha<\frac{1}{1+\left|b_{1}\right|}$ and $\delta \in \mathbb{C}(0<|\delta|<1)$, then the function $\frac{1}{\delta} f(\delta z) \in \mathcal{P}\left(\beta_{1}, \beta_{2}, \beta_{3} ; \lambda\right)$ for $0<|\delta| \leq\left|\delta_{0}(\lambda)\right|$, where $\left|\delta_{0}(\lambda)\right|$ is the smallest positive root of the equation

$$
\begin{aligned}
& \left|\beta_{1}\right| \frac{|\delta|^{2} \sqrt{2\left(1-\alpha-\alpha\left|b_{1}\right|\right)}}{\left(1-|\delta|^{2}\right)^{3 / 2}}+\left|\beta_{2}\right| \frac{|\delta|^{3} \sqrt{6\left(1+3|\delta|^{2}\right)\left(1-\alpha-\alpha\left|b_{1}\right|-2\left|b_{2}\right|^{2}\right)}}{\left(1-|\delta|^{2}\right)^{5 / 2}} \\
& \left|\beta_{3}\right| \frac{|\delta|^{4} \sqrt{48\left(1+8|\delta|^{2}+6|\delta|^{4}\right)\left(1-\alpha-\alpha\left|b_{1}\right|-2\left|b_{2}\right|^{2}-6\left|b_{3}\right|^{2}\right)}}{\left(1-|\delta|^{2}\right)^{7 / 2}} \\
= & \lambda .
\end{aligned}
$$

in $0<|\delta|<1$.

Proof. Since $\frac{z}{f(z)}=1+\sum_{n=1}^{\infty} b_{n} z^{n} \neq 0(z \in \mathcal{U})$ for $f(z) \in \mathcal{H}(\alpha)$, we see that

$$
\frac{z}{\frac{1}{\delta} f(\delta z)}=1+\sum_{n=1}^{\infty} \delta^{n} b_{n} z^{n}
$$

for $0<|\delta|<1$. Thus, to show that $\frac{1}{\delta} f(\delta z) \in \mathcal{P}\left(\beta_{1}, \beta_{2}, \beta_{3} ; \lambda\right)$, from Lemma 2.1 , it is sufficient to prove that

$$
\sum_{n=2}^{\infty} n(n-1)\left(\left|\beta_{1}\right|+(n-2)\left|\beta_{2}\right|+(n-2)(n-3)\left|\beta_{3}\right|\right)|\delta|^{n}\left|b_{n}\right| \leq \lambda .
$$


Applying Cauchy-Schwarz inequality, we note that

$$
\begin{aligned}
& \sum_{n=2}^{\infty} n(n-1)\left(\left|\beta_{1}\right|+(n-2)\left|\beta_{2}\right|+(n-2)(n-3)\left|\beta_{3}\right|\right)|\delta|^{n}\left|b_{n}\right| \\
& \leq\left|\beta_{1}\right|\left(\sum_{n=2}^{\infty} n(n-1)|\delta|^{2 n}\right)^{\frac{1}{2}}\left(\sum_{n=2}^{\infty} n(n-1)\left|b_{n}\right|^{2}\right)^{\frac{1}{2}} \\
&+\left|\beta_{2}\right|\left(\sum_{n=3}^{\infty} n(n-1)(n-2)^{2}|\delta|^{2 n}\right)^{\frac{1}{2}}\left(\sum_{n=3}^{\infty} n(n-1)\left|b_{n}\right|^{2}\right)^{\frac{1}{2}} \\
&+\left|\beta_{3}\right|\left(\sum_{n=4}^{\infty} n(n-1)(n-2)^{2}(n-3)^{2}|\delta|^{2 n}\right)^{\frac{1}{2}}\left(\sum_{n=4}^{\infty} n(n-1)\left|b_{n}\right|^{2}\right)^{\frac{1}{2}} \\
& \leq\left|\beta_{1}\right|\left(\sum_{n=2}^{\infty} n(n-1)|\delta|^{2 n}\right)^{\frac{1}{2}} \sqrt{1-\alpha-\alpha\left|b_{1}\right|} \\
&+\left|\beta_{2}\right|\left(\sum_{n=3}^{\infty} n(n-1)(n-2)^{2}|\delta|^{2 n}\right)^{\frac{1}{2}} \sqrt{1-\alpha-\alpha\left|b_{1}\right|-2\left|b_{2}\right|^{2}} \\
&+\quad\left|\beta_{3}\right|\left(\sum_{n=4}^{\infty} n(n-1)(n-2)^{2}(n-3)^{2}|\delta|^{2 n}\right)^{\frac{1}{2}} \sqrt{1-\alpha-\alpha\left|b_{1}\right|-2\left|b_{2}\right|^{2}-6\left|b_{3}\right|^{2}}
\end{aligned}
$$

We note that

$$
\sum_{n=0}^{\infty} x^{n}=\frac{1}{1-x}, \quad(|x|<1)
$$

thus, we have

$$
\sum_{n=2}^{\infty} n(n-1) x^{n}=\frac{2 x^{2}}{(1-x)^{3}}
$$

Since

$$
\sum_{n=3}^{\infty}(n-2) x^{n-1}=x^{2}\left(\sum_{n=3}^{\infty}(n-2) x^{n-3}\right)=x^{2}\left(\sum_{n=3}^{\infty} x^{n-2}\right)^{\prime}=\frac{x^{2}}{(1-x)^{2}}
$$

we see that

$$
\sum_{n=3}^{\infty}(n-1)(n-2)^{2} x^{n}=x^{3}\left(\frac{x^{2}}{(1-x)^{2}}\right)^{\prime \prime}=\frac{2 x^{3}+4 x^{4}}{(1-x)^{4}} .
$$


and thus, we obtain

$$
\sum_{n=3}^{\infty} n(n-1)(n-2)^{2} x^{n}=\frac{6 x^{3}(1+3 x)}{(1-x)^{5}} .
$$

Furthermore, we have

$$
\begin{aligned}
\sum_{n=4}^{\infty}(n-1)(n-2)^{2}(n-3)^{2} x^{n} & =x^{4}\left(\sum_{n=4}^{\infty}(n-1)(n-2)^{2}(n-3)^{2} x^{n-4}\right) \\
& =x^{4}\left(\sum_{n=4}^{\infty}(n-2)(n-3) x^{n-1}\right)^{\prime \prime \prime}
\end{aligned}
$$

but

$$
\sum_{n=4}^{\infty}(n-2)(n-3) x^{n-1}=x^{3}\left(\sum_{n=4}^{\infty}(n-2)(n-3) x^{n-4}\right)=\frac{2 x^{3}}{(1-x)^{3}}
$$

thus, we have

$$
\sum_{n=4}^{\infty}(n-1)(n-2)^{2}(n-3)^{2} x^{n}=\frac{12 x^{4}+72 x^{5}+36 x^{6}}{(1-x)^{6}}
$$

which yields

$$
\sum_{n=4}^{\infty} n(n-1)(n-2)^{2}(n-3)^{2} x^{n}=\frac{48 x^{4}\left(1+8 x+6 x^{2}\right)}{(1-x)^{7}} .
$$

Therefore, from (2.3)- (2.6) with $|\delta|^{2}=x$, we obtain

$$
\begin{aligned}
& \sum_{n=2}^{\infty} n(n-1)\left(\left|\beta_{1}\right|+(n-2)\left|\beta_{2}\right|+(n-2)(n-3)\left|\beta_{3}\right|\right)|\delta|^{n}\left|b_{n}\right| \\
\leq & \left|\beta_{1}\right| \frac{|\delta|^{2} \sqrt{2\left(1-\alpha-\alpha\left|b_{1}\right|\right)}}{\left(1-|\delta|^{2}\right)^{3 / 2}}+\left|\beta_{2}\right| \frac{|\delta|^{3} \sqrt{\left(6+18|\delta|^{2}\right)\left(1-\alpha-\alpha\left|b_{1}\right|-2\left|b_{2}\right|^{2}\right)}}{\left(1-|\delta|^{2}\right)^{5 / 2}} \\
& \left|\beta_{3}\right| \frac{|\delta|^{4} \sqrt{48\left(1+8|\delta|^{2}+6|\delta|^{4}\right)\left(1-\alpha-\alpha\left|b_{1}\right|-2\left|b_{2}\right|^{2}-6\left|b_{3}\right|^{2}\right)}}{\left(1-|\delta|^{2}\right)^{7 / 2}}
\end{aligned}
$$


Now, let us consider the complex number $\delta(0<|\delta|<1)$ such that

$$
\begin{aligned}
& \left|\beta_{1}\right| \frac{|\delta|^{2} \sqrt{2(1-\alpha)}}{\left(1-|\delta|^{2}\right)^{3 / 2}}+\left|\beta_{2}\right| \frac{|\delta|^{3} \sqrt{6\left(1+3|\delta|^{2}\right)\left(1-\alpha-2\left|b_{2}\right|^{2}\right)}}{\left(1-|\delta|^{2}\right)^{5 / 2}} \\
& \left|\beta_{3}\right| \frac{|\delta|^{4} \sqrt{48\left(1+8|\delta|^{2}+6|\delta|^{4}\right)\left(1-\alpha-2\left|b_{2}\right|^{2}-6\left|b_{3}\right|^{2}\right)}}{\left(1-|\delta|^{2}\right)^{7 / 2}} \\
= & \lambda .
\end{aligned}
$$

If we define the function $h(|\delta|)$ by

$$
\begin{aligned}
h(|\delta|)= & \left|\beta_{1}\right||\delta|^{2}\left(1-|\delta|^{2}\right)^{2} \sqrt{2\left(1-\alpha-\alpha\left|b_{1}\right|\right)} \\
& +\left|\beta_{2}\right||\delta|^{3}\left(1-|\delta|^{2}\right) \sqrt{\left(6+18|\delta|^{2}\right)\left(1-\alpha-\alpha\left|b_{1}\right|-2\left|b_{2}\right|^{2}\right)} \\
& +\left|\beta_{3}\right||\delta|^{4} \sqrt{48\left(1+8|\delta|^{2}+6|\delta|^{4}\right)\left(1-\alpha-\alpha\left|b_{1}\right|-2\left|b_{2}\right|^{2}-6\left|b_{3}\right|^{2}\right)} \\
& -\lambda\left(1-|\delta|^{2}\right)^{7 / 2},
\end{aligned}
$$

then we have

$h(0)=-\lambda<0$ and $h(1)=\left|\beta_{3}\right| \sqrt{720\left(1-\alpha-\alpha\left|b_{1}\right|-2\left|b_{2}\right|^{2}-6\left|b_{3}\right|^{2}\right)}>0$. This means that there exists some $\delta_{0}$ such that $h\left(\left|\delta_{0}\right|\right)=0\left(0<\left|\delta_{0}\right|<1\right)$. This completes the proof of the theorem.

\section{Acknowledgement}

The authors wishes to thank the referees for his/her useful comments and suggestions.

\section{References}

[1] H. Kobashi, K. Kuroki, H. Shiraishi, and S. Owa, Radius problems of certain analytic functions, Internat. J. Open Problems Complex Anal., 1, (2009), 8-12.

[2] H. Kobashi, K. Kuroki, and S. Owa, Notes on radius problems of certain univalent functions, General Mathematics, 17, No. 4 (2009), 5-12.

[3] H. Kobashi, H. Shiraishi, and S. Owa, Radius problems of certain starlike functions, J. Adv. Math. Studies, 3, No. 2 (2010), 57-64. 
Basem Aref Frasin

$\mathrm{Al}$ al-Bayt University, Faculty of Sciences,

Department of Mathematics

P.O. Box 130095, Mafraq 25113, Jordan

E-mail: bafrasin@yahoo.com

Jin-Lin Liu

Department of Mathematics, Yangzhou University,

Yangzhou, 225002, P.R.China

E-mail: jlliu@yzu.edu.cn

Received: 16.07.2012

Accepted: 4.10.2012 